\title{
Möglichkeiten neuer Informationsflüsse im OP-Bereich
}

\author{
Uwe Kleineidam, Fritz Manz
}

\section{Zusammenfassung}

Die zunehmenden fachlichen Vorausreichen machen die Notwendigkeit von sowohl traditionellen aber auch neuen Formen von Informationswegen notwendig. Die traditionellen Ordner und Heftverfahren sind aufgrund ihrer schlechten Zugriffsmöglichkeiten im OP-Saal eher als Dokument der Standardfestschreibung geeignet. Die interaktive Suche von setzungen und Kenntnisse in OP-Be-

gerade benötigten operationsrelevanten Informationen scheitert bei traditionellen Verfahren (Infobroschüren, Aufzeichnungen etc.) an der dicht gedrängten Zeit innerhalb der OP-Abläufe. Die Integrierung einer EDV-Lösung in Informationsabläufe bietet derzeit den höchst möglichen Komfort. Die interessant gestalteten Themenblöcke umfassen Texte, Bilder ja sogar Videosequenzen.

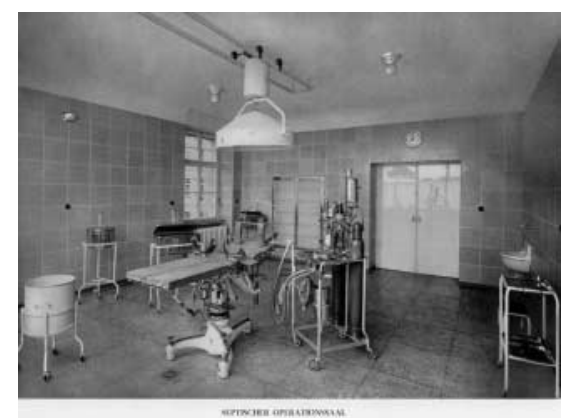

Abb.1 Septischer Operationssaal 1927.

Da der Einsatzbereich und das Spektrum der operativen Fächer deutlich kleiner waren als in den heute bestehenden „Multifunktions- OP's“, konnten die erworbenen Kenntnisse schnell erlernt, und in der täglichen Arbeit umgesetzt werden.

Ein kontinuierlicher Fortbildungsprozess, welcher gerade bei Betrieben mit OPWeiterbildungen oder OTA-Schulen zwingend gefordert werden muss, war zu diesem Zeitpunkt weder planerisch noch in einer bestimmten Organisationsform festgehalten.

OP-JOURNAL 2003; 19: 238-242

(c) Georg Thieme Verlag Stuttgart . New York

\section{Neue Anforderungen im OP-Bereich}

Der rasante Fortschritt in den unterschiedlichen operativen Fächern im OPBereich lässt aus heutiger Sicht eine meist auf freiwilliger Basis funktionierende Informationsweitergabe nicht mehr $\mathrm{zu}$, und fordert konkrete Aus- und Weiterbildungskonzepte zur fortwährenden Vertiefung und Neuerwerb von medizinischem Wissen.

Defizite im Kenntnisstand ergeben sich weniger durch Fehlen von Informationsmaterial, sondern auch durch Art und Methodik wie Informationen angeboten werden können.

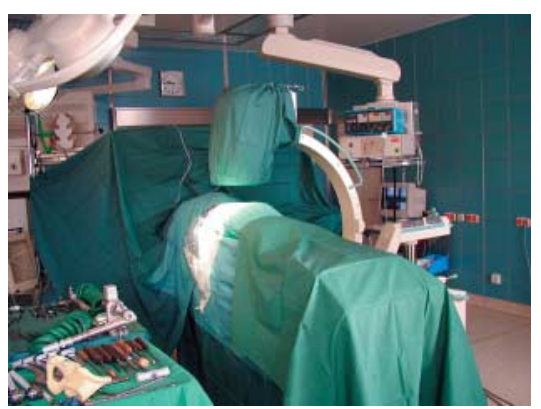

Abb. 2 Moderner OP-Saal.

Große Bedeutung hat dieses Problem in Fortbildungsstätten, wie OP-Fachweiterbildung oder OTA-Schule.

Neben der personellen und logistischen Ausrüstung einer Weiterbildungsstätte, sollte das Informationsangebot nicht darauf beschränkt sein, lediglich den WBTeilnehmer zu erreichen, sondern auch jeden Mitarbeiter des Stammpersonals. Ziel ist es, in der täglichen Arbeit ein sicheres und leicht zu bedienendes Modul einzusetzen, welches dem Mitarbeiter ermöglicht, schnell und am Arbeitsplatz die gewünschten Informationen zu erhalten.

Dies war und ist die Grundidee, einen neuen Weg des Kommunikationsflusses aufzubauen.

\section{Traditionelle Informationswege}

Jeder von uns kennt sie wohl, die gut behüteten Ordner, in welchem Broschüren, Anweisungen, Standards usw. abgeheftet worden sind.

So gut diese Fixierung von Wissen war, so schwierig ist es, einen Teil der Informationen für den täglichen Gebrauch daraus wieder abzurufen.

Bei der permanenten Zunahme an Spezialisierung in den operativen Fächern, nimmt eine auf Aktenführung beruhende Dokumentation gewaltige Ausmaße an. 


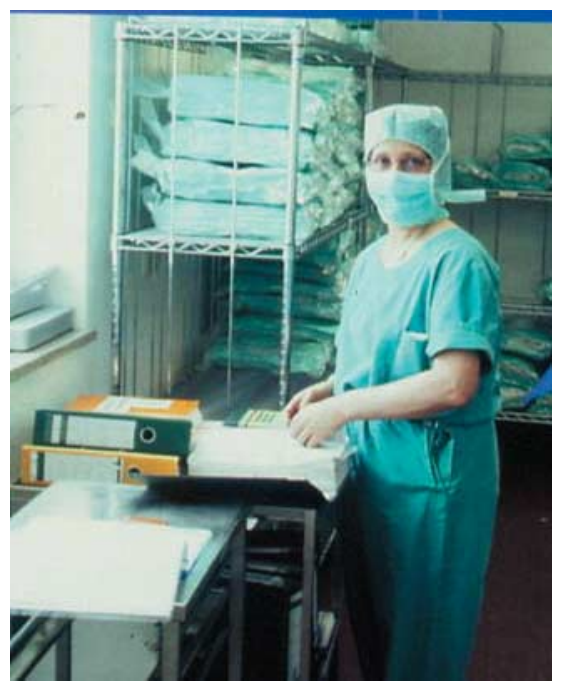

Abb.3 Gesammelte Unterlagen in Ordnern.

Ebenso ist die Notwendigkeit der fortwährenden Aktualisierung der Dokumente kaum vollziehbar.

Für den Mitarbeiter ist es daher oftmals nicht sicher, ob das, was er gerade gelesen hat noch Gültigkeit besitzt, oder schon überholt oder verändert ist.

Ich möchte jedoch darauf hinweisen, dass eine Dokumentation in dieser Weise besser ist als keine.

Die Diskrepanz, welche sich in diesen Systemen aber zeigt, scheint nach heutigen Qualitätsanforderungen dem Anspruch von umfassender Information nicht mehr gerecht zu werden.

\section{EDV-Abteilungen}

Die Möglichkeit zu flächendeckenden Informationen, ist aus heutiger Sicht durch Einführung von vernetzten EDV-Lösungen grundsätzlich möglich.

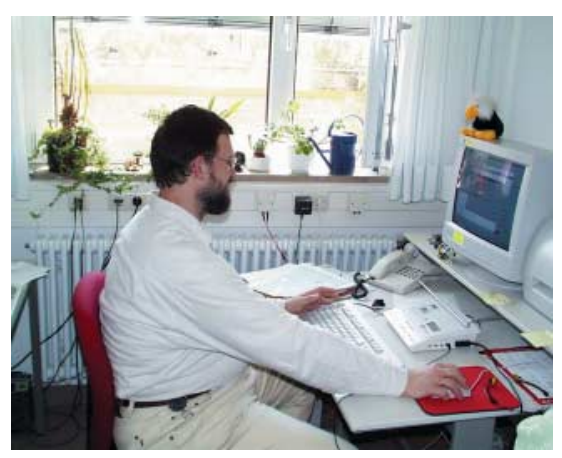

Abb.4 Enge Zusammenarbeit mit der EDVAbteilung.

Die Strukturen der Krankenhausinformationssysteme sind geeignet, den Wissenstransport in allen Bereichen des Krankenhauses zu ermöglichen.

Auf der „Datenautobahn“, welche zunächst von den Verwaltungen eingefordert wurde, um Informationen aus dem OP- und anderen Bereich abzubilden, ermöglicht es auch, ebenfalls eine komplexe Struktur der Bildungsinformation in den bestehenden Systemen aufzubauen.

Die enge Zusammenarbeit mit der EDVAbteilung ist Basis und zwingende Voraussetzung von derartigen Systemen.

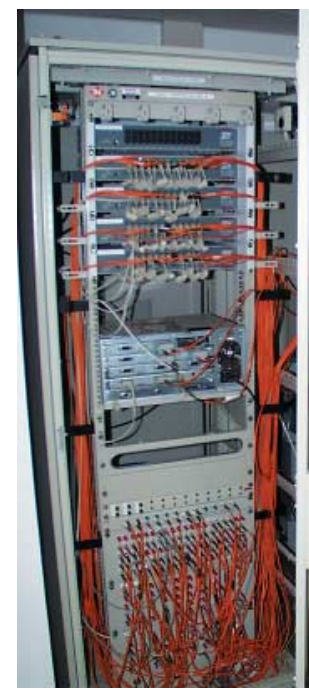

Abb. 5 Hochtechnisiert teme.

Betrachtet man den Datentransfer aus und in den OP-Bereich, so wird klar, welcher Aufwand für Hard- und Software aufgewendet werden muss.

Einige Beispiele verdeutlichen den hohen Datenfluss:

- Patientenverwaltungssystem

- OP-Dokumentationssysstem

- Anästhesiesystem

- Zeiterfassungssystem

- Lagerwirtschaftssystem

- Dienstplanungssystem

- Arztbrief- und Verschlüsselungssystem

- usw.

Längst ist die Diskussion über die Frage, ob das Pflege- oder ärztl. Personal EDV benutzen soll, oder kann, in Richtung-Anwendung solcher Systeme - entschieden.

Sicherlich arbeitet jede Klinik in der EDVund Vernetzungsproblematik an einem unterschiedlichen Level.
Festzustellen ist jedoch, dass scheinbar niemand mehr an derartigen Systemen vorbei kommt.

Für mich stellte sich nun die Frage, ob und wie wir in der Abteilung diese Systeme einbinden und Nutzen daraus ziehen können.

Die Hard- und Software war vorhanden. Was fehlte, war die Idee, diese jetzt als Medium zum Transfer von Informationen im Weiterbildungssektor zu nutzen.

\section{Grundidee und Lösungen}

Der Grundgedanke war, die Information dort anzubieten, wo sie tatsächlich auch gebraucht wird, dies ist unbestritten im OP-Saal.

Meiner Einschätzung nach wäre der Mitarbeiter („Springer“, Instrumenteur, der Krankenpflegeschüler, die OTA, der Weiterbildungsteilnehmer) froh, ein Hilfsmittel zu besitzen, welches auf leicht verständlichen Bedienoberflächen mit sinnvollen Wortkürzeln zugreifen könnte.

Andererseits muss ausgeschlossen sein, dass durch Bedienungsfehler oder durch Unachtsamkeit die Inhalte der Dokumente ständig verändert oder gar zerstört wurden.

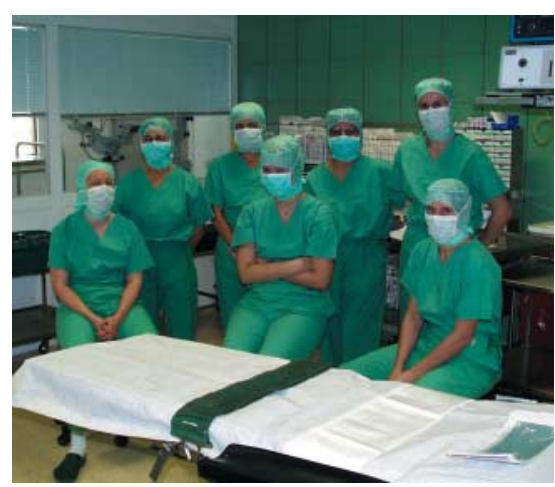

Abb. 6 Einsatz der Weiterbildungsteilnehmer.

Die leichte Bedienbarkeit sollte Garant für die Akzeptanz des Systems sein. Ebenfalls die Möglichkeit, Texte und Bilder bei Bedarf ausdrucken zu können.

\section{Forderung}

Der Umfang der Informationen und die Bedienung dürfen zu keiner Beeinträchtigung anderer laufender Systeme führen. 


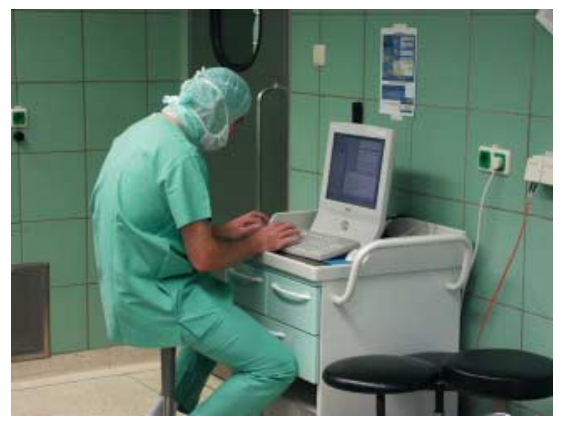

Abb. 7 Arbeiten am PC im OP-Saal.

Auf der Basis dieser Kriterien kam die Konvertierung der bestehenden Texte, Film, Bilder in HTML-Formaten zum Tragen (HTML).

Der in der Standardinstallation des PC zu öffnende Internet-Explorer stellte die Bedieneroberfläche zur Verfügung. (zzt. Explorer 5.5)

Da den meisten Mitarbeitern die Arbeit im Internet im privaten Bereich schon bekannt war, musste dieser Punkt nur wenigen extra erläutert werden.

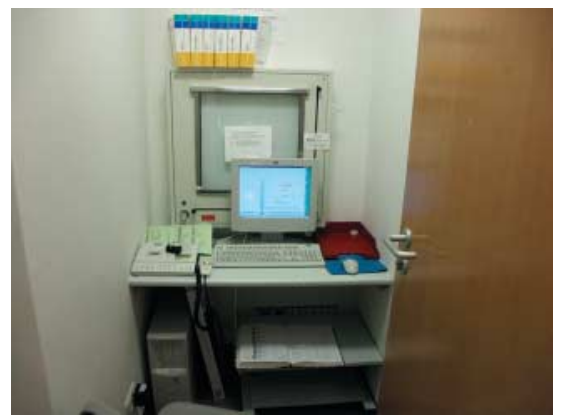

Abb. 8 Arbeitsplatz Kodierung OP.

Durch die Vernetzung und Vereinheitlichung der Systeme war es nun möglich, praktisch an jeden Ort im Haus Informationen zu senden und abzurufen.

\section{Lösungsbeispiel}

Auf jedem Rechner ist in der Standardinstallation der Internet Explorer installiert. $\mathrm{Zu}$ erreichen ist hierüber

- Internetzugang WWW.

- und Intra-Net,

welches innerhalb des Krankenhauses seinen Einsatz findet.

Das von mir beschriebene Informationssystem läuft im Intra-Net.

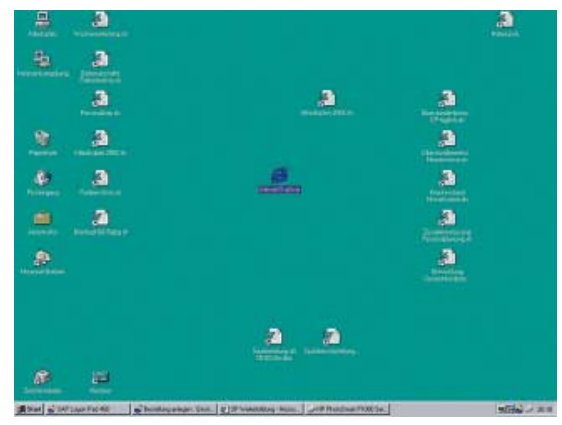

Abb.9 Startmaske auf dem Bildschirm.

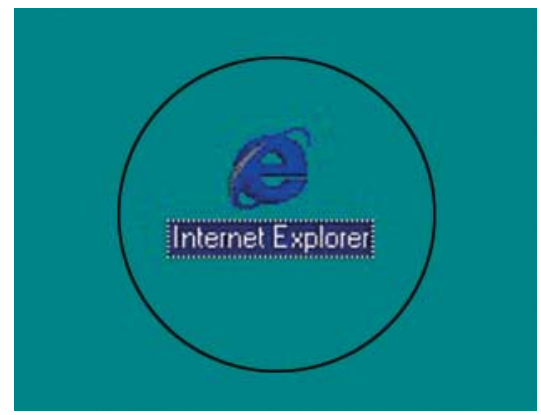

Abb.10 Zugang der Information über Internet-Explorer.

Nach Anklicken auf die Explorer-Icons öffnet sich eine Maske, welche die verschiedenen Informationskanäle anzeigt.

Durch Anklicken der Button „Abteilungen“ führt einen der Bildschirm in die Rubrik der verschiedenen Abteilungen.

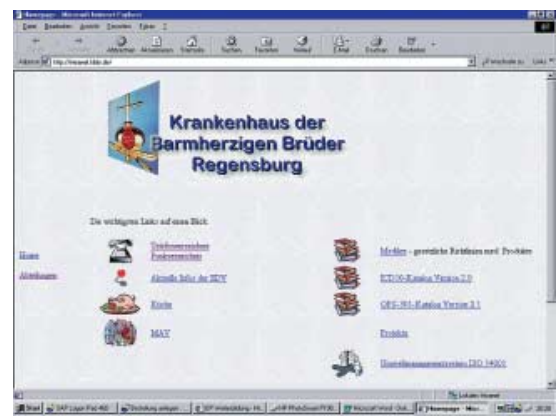

Abb.11 Übersichtsmaske der verschiedenen Anbieter von Informationen im Krankenhaus.

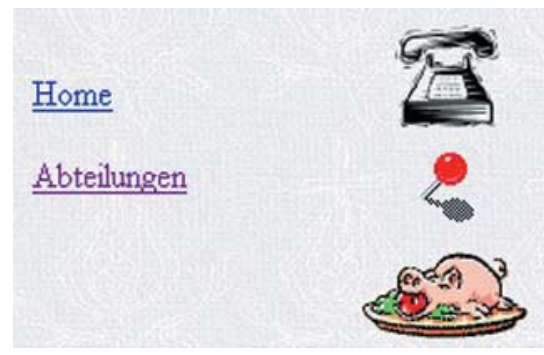

Abb.12 Leicht verständliche Buttons erleichtern die Suche.

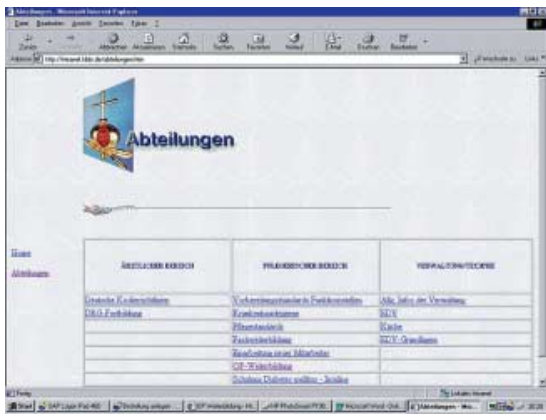

Abb.13 Maske der anbietenden Bereiche.

\begin{tabular}{|l|}
\hline Vorbereitungsstandards Funktionsstellen \\
\hline Krankenhaushygiene \\
\hline Pflegestandards \\
\hline Fachweiterbildung \\
\hline Einarbeitung neuer Mitarbeiter \\
\hline OP-Weiterbildung \\
\hline Schulung Diabetes mellitus - Insuline \\
\hline
\end{tabular}

Abb.14 Übersichtlicher Zugang durch Strukturierung.

Die OP-Weiterbildung befindet sich im Pflegerischen Bereich.

Durch Anklicken des Button „OP-Weiterbildung “ wird man in das Eröffnungsmenü der OP-Weiterbildung geführt.

Hier werden die an der Erstellung des Programms beteiligten Mitarbeiter kurz vorgestellt, und über einen Einleitungssatz wird das Ausbildungsziel erläutert. Auf der linken Laufleiste stehen die unterschiedlichen Fachabteilungen aufgegliedert.

Ebenso besteht die Möglichkeit, per Mausklick mit den produktbeteiligten Mitarbeitern in informellen Kontakt zu treten.

Klickt man mit der Maus nun zum Bespiel in der linken Laufleiste auf das Button „Unfallchirurgie“, so öffnet sich eine weitere Maske, welche durch grobe Einteilung der Topografie des Menschen, und rechts davon die dazu passenden Themen untergliedert.

Nach Selektion des gewünschten Themas und durch Anklicken mit der „Maus“ öffnet sich der komplette Inhalt des Dokumentes.

Neben Texten, welche den Standard von Instrumenten, Abdeckungen, Lagerungen, Nahtmaterialien, Anatomie usw. 


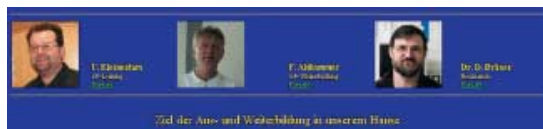

Abb.15 Eröffnungsmaske zur OP-Weiterbildung.

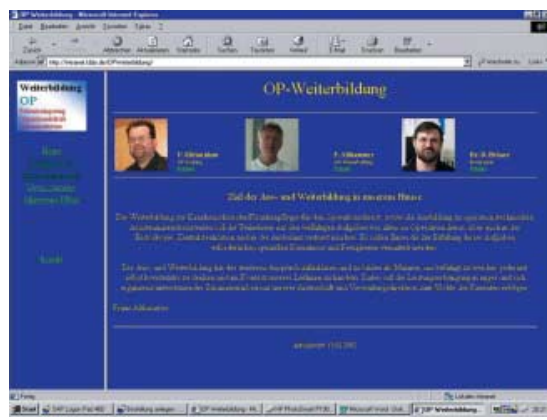

Abb.16 Links zu den Betreuern des Systems.

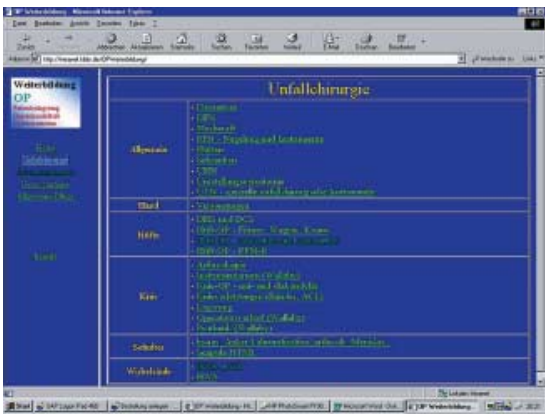

Abb.17 Feinmaske der gewählten Fachabteilung.

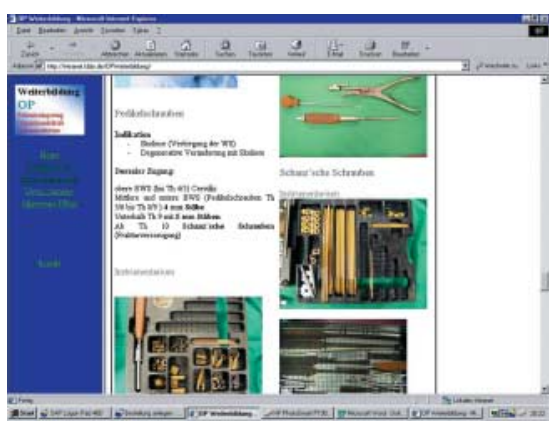

Abb.18 Geöffnetes Dokument.

zeigt, sind entsprechende bildhafte Darstellungen eingebunden, welches dem Text zugeordnet zum besseren Verstehen verhelfen soll.

Ein Zurückfinden in die Hauptmaske ist jederzeit möglich. Ein Wechsel in eine andere Fachabteilung ebenfalls. Um bessere Übersicht über das schon gelesene Dokument zu haben, werden geöffnete Dokumente farblich unterlegt.

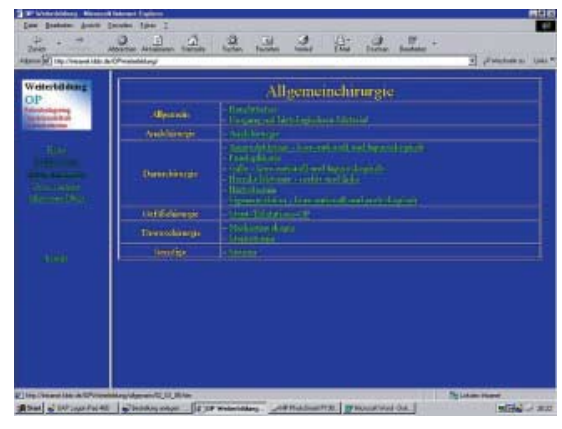

Abb.19 Wechsel in ein anderes Fachgebiet.

Der Mitarbeiter kann sowohl mit der Maus innerhalb des Protokolls auf- und abfahren oder mit der Scroll-Leiste seitenweise sich durch das Dokument lesen.

$\mathrm{Zu}$ keiner Zeit, selbst wenn Texte angeklickt oder unterlegt werden, kann eine Veränderung des Dokumentes durchgeführt werden.

Ein Ausdruck des gerade geöffneten Dokumentes ist dagegen möglich.
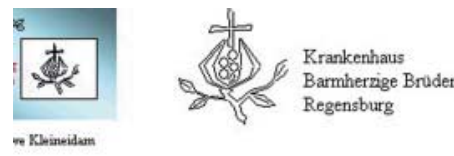

Whate therateiture am 2001-10-21

sektion

Überblick über den Dickdarm (schematisch nach Röntgenbildern)

Abb. 20 Hinweis auf aktuelle Überarbeitungen im neuen System.

Für den Mitarbeiter hilfreich ist der Hinweis zur letzten Überarbeitung des aktuell geöffneten Dokumentes. Dieser Hinweis befindet sich in der Kopfleiste jedes Dokumentes.

Sollten Fragen oder Verbesserungen auffallen oder gewünscht werden, so kann der Mitarbeiter per Mausklick seine Fragestellung oder Wünsche dem Autor, der Schulleitung oder der EDV-Abteilung schriftlich mitteilen.

Als zusätzliche multimediale Möglichkeit werden bei interessanten Operationsabschnitten Videosequenzen angeboten.

Für den Mitarbeiter werden diese Videoclips farblich angezeigt, und ein Mottobild zum Video, am Dokument sichtbar.
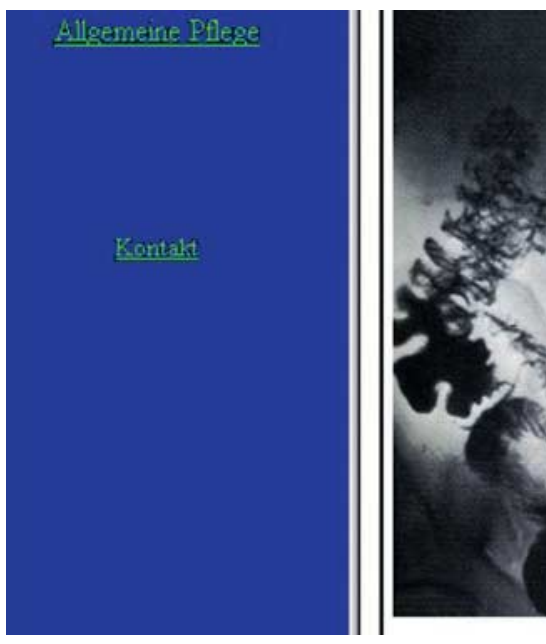

Abb. 21 Fragen, Verbesserungen etc. können über Kontakt direkt mitgeteilt werden.

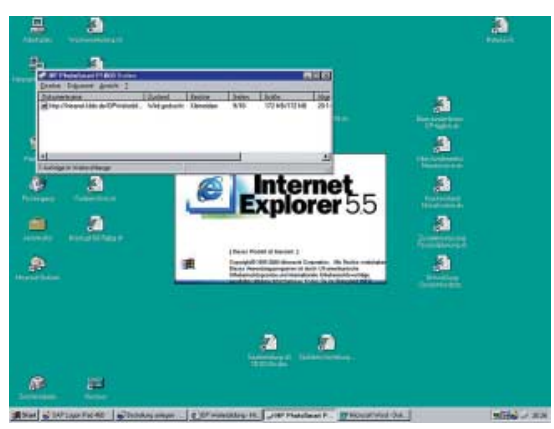

Abb. 22 Der zentral angeschlossene Drucker kann jederzeit einen Konzeptausdruck erstellen.

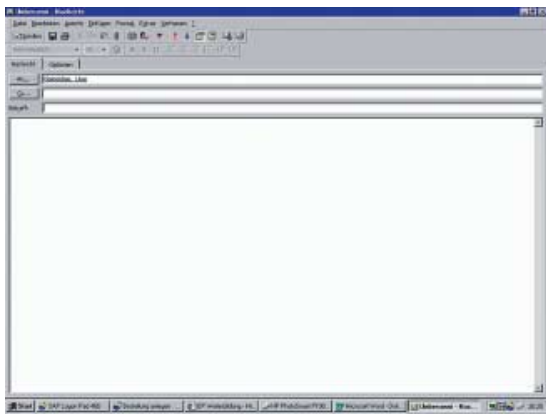

Abb. 23 Interaktiver Kontakt zum Autor, EDV-Abteilung oder Schulleitung möglich.

Durch Anklicken der farblich hinterlegten Videoclips öffnet sich der ebenfalls standardmäßig installierte Medienplayer, welcher nach kurzer Ladephase den Videofilm selbstständig abspielt.

Der Mitarbeiter kann zu jeder Zeit den Film anhalten, zurückspulen, wiederholen oder die Standardgröße des Anzeigeformates einstellen. 


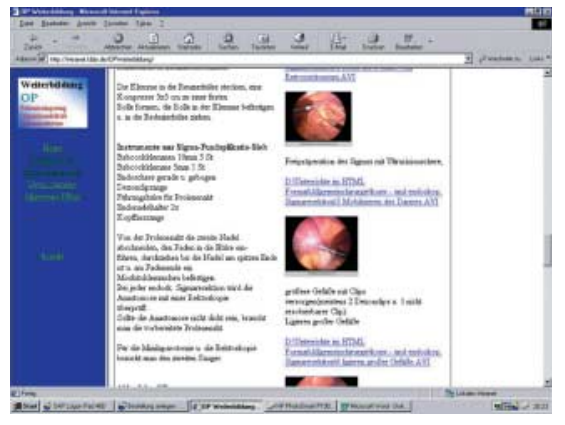

Abb.24 Videosequenzen innerhalb eines Dokumentes.

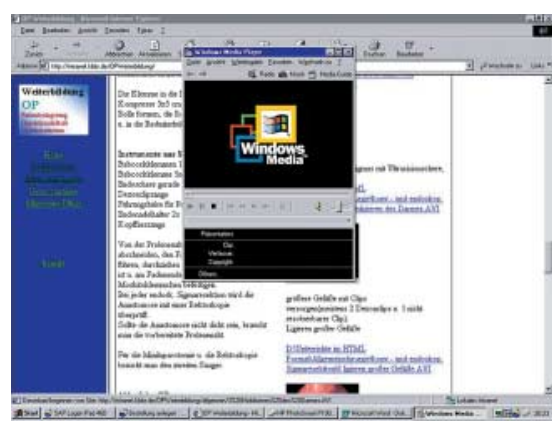

Abb. 25 Medienplayer öffnet automatisch den Videoclip.

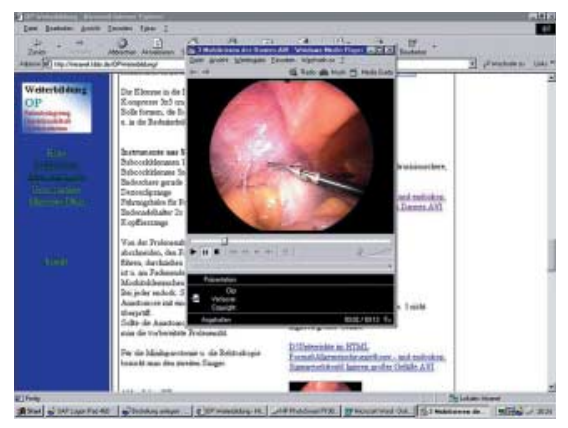

Abb. 26 Videosequenzen komplettieren die Erläuterungen.
Der Mitarbeiter gewinnt somit ein völlig neues Informationserlebnis. Konzentrierte Informationen zu den gewünschten Dokumenten.

Die Komplettierung durch bewegte Arbeitsabläufe macht schwierige Erläuterungen leicht verständlich und interessant.

Dieses System der Informationsweitergabe auf der Basis von Unterrichtsmaterial, Absprachen mit den Chirurgen, echten Operationsabläufen und Standards der Abteilung ist auf fortwährende Entwicklung ausgelegt.

Je mehr Prozesse dokumentiert werden, umso umfangreicher und kompletter wird die Datenbank.

So gesehen, ist der angestoßene Prozess auf Jahre hin weiter entwicklungsfähig.

Da versucht wird, sowohl zeitgemäße Technik einzusetzen, aber auch die einzelnen Präsentationen interessant zu gestalten, tritt die Akzeptanzfrage für Nutzer und Betreiber nicht auf.

Neue, kreative Geister werden geweckt und führen zu erstaunlichem Ideenreichtum und motivierten Mitarbeitern.
Uwe Kleineidam

OP-Leitung

Fritz Manz Pflegedirektor

(Projektbegleitung)

Krankenhaus der Barmherzigen Brüder Prüfeninger Str. 86

D-93049 Regensburg 